\title{
Review Article \\ Formation, Energetics, and Electronic Properties of Graphene Monolayer and Bilayer Doped with Heteroatoms
}

\author{
Yoshitaka Fujimoto \\ Department of Physics, Tokyo Institute of Technology, 2-12-1 Oh-okayama, Meguro-ku, Tokyo 152-8551, Japan \\ Correspondence should be addressed to Yoshitaka Fujimoto; fujimoto@stat.phys.titech.ac.jp
}

Received 28 April 2015; Revised 9 July 2015; Accepted 26 July 2015

Academic Editor: Da-Ren Hang

Copyright ( 2015 Yoshitaka Fujimoto. This is an open access article distributed under the Creative Commons Attribution License, which permits unrestricted use, distribution, and reproduction in any medium, provided the original work is properly cited.

\begin{abstract}
Doping with heteroatoms is one of the most effective methods to tailor the electronic properties of carbon nanomaterials such as graphene and carbon nanotubes, and such nanomaterials doped with heteroatom dopants might therefore provide not only new physical and chemical properties but also novel nanoelectronics/optoelectronics device applications. The boron and nitrogen are neighboring elements to carbon in the periodic table, and they are considered to be good dopants for carbon nanomaterials. We here review the recent work of boron and nitrogen doping effects into graphene monolayer as well as bilayer on the basis of the first-principles electronic structure calculations in the framework of the density-functional theory. We show the energetics and the electronic properties of boron and nitrogen defects in graphene monolayer and bilayer. As for the nitrogen doping, we further discuss the stabilities, the growth processes, and the electronic properties associated with the plausible nitrogen defect formation in graphene which is suggested by experimental observations.
\end{abstract}

\section{Introduction}

Ever since its discovery in 2004, graphene, a single-layer sheet of hexagonal carbon arrangement, has attracted a lot of interest because it shows unique mechanical and electronic properties [1-5]. Graphene exhibits extremely elastic property and strong stiffness [5], the electrons in graphene behave as massless Dirac fermions [2], and graphene also possesses the enormously high carrier mobility [3]. It has been also reported that several exotic quantized phenomena have emerged [6-9]. Carbon nanotubes have also received much interest since its discovery in 1991 [10]. The single-walled carbon nanotubes exhibit the metallic and semiconducting properties, depending on how a graphene sheet is rolled up into the cylindrical shape, that is, the spiral conformation of the nanotubes [11]. Furthermore, carbon nanotubes show ballistic transport properties even at room temperature [12] and the sustaining current density of metallic nanotubes is larger by 1000 times than that of copper [13].

One of the most effective methods to tailor electronic properties of graphene and carbon nanotubes is to dope heteroatoms into the hexagonal carbon network. The boron- (B-) and nitrogen- $(\mathrm{N}-)$ doped graphene and carbon nanotubes have been synthesized so far through chemical vapor deposition and electrothermal reactions [14-31]. The $p$-type fieldeffect transistors (FETs) are successfully produced by using the B-doped graphene, and it is shown that the carrier mobility and the band gaps vary depending on the Bdopant concentration $[14,15]$. It is also reported that $\mathrm{B}$-doped graphene behaves as efficient oxygen reduction reaction (ORR) catalysts with alternative ORR active sites [16]. On the other hand, recent experimental measurements of X-ray photoelectron spectroscopy on $\mathrm{N}$-doped graphene as well as $\mathrm{N}$-doped carbon nanotube have revealed the existence of two major $\mathrm{N}$-defect bonding configurations. One is the substitutional $\mathrm{N}$ defect where the $\mathrm{C}$ atom is simply replaced by the $\mathrm{N}$ atom and the other is the pyridine-type $\mathrm{N}$ defects where the $\mathrm{N}$ atoms are located around vacancies [17-24]. It has been reported theoretically that the structural variety of $\mathrm{N}$ defects in graphene and carbon nanotubes provides various electronic properties such as $p$-type and $n$-type conduction properties depending on how atomic configurations of $\mathrm{N}$ defects are formed [22, 25, 26]. Actually, the N-doped CNTs are shown experimentally to have $p$-type transport properties, while the $\mathrm{N}$-doped graphene has produced $n$ type doping properties $[18,22]$. The $\mathrm{N}$-doped graphene 
also enhances the electrostatic capacitance compared with undoped graphene $[32,33]$. It is also reported that the pyridine-type $\mathrm{N}$-doped graphene adsorbed with transition metals might provide hydrogen storages and acts as chemical reaction centers [34-36]. Thus, carbon nanomaterials doped with $\mathrm{B}$ and $\mathrm{N}$ atoms would offer rich electronic properties and are expected to be useful as potential device materials in nextgeneration nanoelectronics applications [37-43].

The purpose of this review is to provide the first-principles density-functional study on atomic structures, energetics, and electronic properties of boron and nitrogen defects in graphene monolayer as well as bilayer. Section 2 discusses boron and nitrogen doping effects on atomic configurations and stabilities of monolayer and bilayer graphenes. In Section 3, we show the electronic structures of $\mathrm{B}$ and $\mathrm{N}$ doped graphenes and demonstrate the scanning tunneling microscopy (STM) images of B and $\mathrm{N}$ defects in graphene to identify those defects. Finally, we summarize this paper in Section 4.

\section{Energetics and Structure}

In this section, the atomic structures and the energetics of boron and nitrogen defects in monolayer and bilayer graphenes are reported. First, the doping effects of substitutional $\mathrm{B}$ and $\mathrm{N}$ dopants, where $\mathrm{C}$ atom is simply replaced by $\mathrm{B}$ and $\mathrm{N}$ atoms, into monolayer as well as bilayer graphenes are studied, and then the nitrogen-vacancy complex defects in graphene are also examined to identify the possible configurations among various types of the pyridine-type defects.

To discuss the energetics of $\mathrm{B}$ - and $\mathrm{N}$-doped monolayer as well as bilayer graphenes, the formation energy $E_{f}$ is defined by

$$
E_{f}=E_{\text {tot }}-m_{\mathrm{C}} \mu_{\mathrm{C}}-m_{\mathrm{B} \text { or N }} \mu_{\mathrm{B} \text { or N }}
$$

where $E_{\text {tot }}$ is the total energies of the B- or $\mathrm{N}$-doped monolayer graphene and bilayer graphene with $A A, A B 1$, and $A B 2$ stacking patterns, $m_{\mathrm{C}}$ is the number of $\mathrm{C}$ atoms in a supercell, and $\mu_{\mathrm{C}}, \mu_{\mathrm{B}}$, and $\mu_{\mathrm{N}}$ are the chemical potentials of pristine monolayer graphene, pristine bilayer graphene with corresponding $A A$ or $A B$ stacking pattern, $\alpha$-boron crystal, and $\mathrm{N}_{2}$ molecule, respectively.

2.1. B and N Defects in Monolayer and Bilayer Graphenes. Before examining the atomic structures of B- and N-doped graphenes, the structural properties of undoped pristine graphene are shown (Figure 1). The calculated lattice constant of the pristine graphene is $2.44 \AA$, which is in good agreement with the experimental value of $2.46 \AA$.

We next study the structural properties of substitutional boron and nitrogen defects where one $\mathrm{C}$ atom is replaced by the $\mathrm{B}$ and the $\mathrm{N}$ atoms. The $\mathrm{B}-\mathrm{C}$ bond length and the $\mathrm{C}-\mathrm{N}$ one in the substitutionally $\mathrm{B}$ - and $\mathrm{N}$-doped graphenes are found to be $1.47 \AA$ and $1.40 \AA$, respectively. The B-C bond length is much longer than the calculated C-C bond length of $1.41 \AA$ in the pristine graphene, whereas the $\mathrm{C}-\mathrm{N}$ bond length is slightly shorter than the $\mathrm{C}-\mathrm{C}$ bond length of the pristine graphene. In

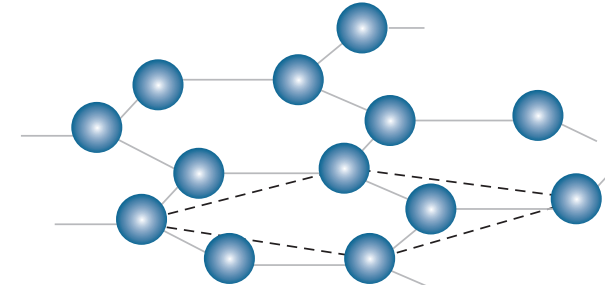

FIgURE 1: Schematic view of monolayer graphene. The dotted line denotes the unit cell.

addition, the $\mathrm{B}$ and $\mathrm{N}$ atoms seem to reside in a planar $s p^{2}$ bonding configuration.

We here examine atomic structures of pristine bilayer graphene. For stacking patterns of bilayer graphene, we use two types of bilayer graphene: $A A$ and $A B$ stacking (see Figure 2). The $A B$ stacking as well as the $A A$ stacking patterns of undoped bilayer graphenes is experimentally observed [44, 45]. The calculated interlayer distances of $A A$ and $A B$ stacking are $3.60 \AA$ and $3.33 \AA$, respectively. In addition, the calculated interlayer distance of the $A B$-stacked bilayer graphene is similar to the interlayer distance of bulk graphite observed experimentally [46]. The bond lengths between two $C$ atoms in a graphene planar sheet are $1.41 \AA$ for both $A A$ and $A B$ stacking patterns as in the case of monolayer graphene.

We also examine atomic structures of $\mathrm{B}$ - and $\mathrm{N}$-doped bilayer graphenes. For $A B$-stacking pattern, we consider two types of $\mathrm{B}(\mathrm{N}-)$ doped bilayer graphene as depicted in Figure 2. One is that the $\mathrm{C}$ atom on top of the $\mathrm{C}$ atom in the lower graphene layer is replaced with a dopant, which is referred to $A B 1$ stacking, and the other is that the $\mathrm{C}$ atom on top of the center of a hexagon in the lower graphene layer is replaced with a dopant, which is referred to $A B 2$ stacking. The interlayer distances of $\mathrm{B}$-doped as well as $\mathrm{N}$-doped bilayer graphenes are $3.60 \AA, 3.33 \AA$, and $3.33 \AA$ for $A A, A B 1$, and $A B 2$ stacking patterns, respectively. The $\mathrm{B}-\mathrm{C}$ bond and the $\mathrm{C}-\mathrm{N}$ bond lengths in the doped graphene planar sheet are $1.47 \AA$ and $1.40 \AA$, respectively, for $A A, A B 1$, and $A B 2$ stacking, and both $\mathrm{B}$ and $\mathrm{N}$ atoms are in the planar sheet. The B-C bond length is much longer than the $\mathrm{C}-\mathrm{C}$ bond length in a pristine bilayer graphene, while the $\mathrm{C}-\mathrm{N}$ bond length is slightly shorter than the $\mathrm{C}-\mathrm{C}$ bond length in a pristine one. They are almost the same value as B-C bond length of the Bdoped monolayer graphene and the $\mathrm{C}-\mathrm{N}$ bond length in $\mathrm{N}$ doped one.

We now study the stabilities of $\mathrm{B}$ - and $\mathrm{N}$-doped graphenes. To discuss the energetics of $\mathrm{B}$ - and $\mathrm{N}$-doped monolayer and bilayer graphenes, we show the results of the formation energy $E_{f}$ defined by (1). The formation energies of the substitutionally $\mathrm{B}$ - and $\mathrm{N}$-doped monolayer graphenes are $1.42 \mathrm{eV}$ and $0.32 \mathrm{eV}$, respectively. The formation energies of $A A$-stacked bilayer graphene doped with $\mathrm{B}$ and $\mathrm{N}$ atoms are $1.355 \mathrm{eV}$ and $0.240 \mathrm{eV}$, respectively, which are the smallest among doping sites into three kinds of stacking patterns $A A, A B 1$, and $A B 2$ for both $\mathrm{B}$-doped and $\mathrm{N}$-doped cases. In addition, the $\mathrm{B}$ - and $\mathrm{N}$-doping into $A A$-stacked bilayer graphene are energetically favorable rather than those into monolayer graphene. 


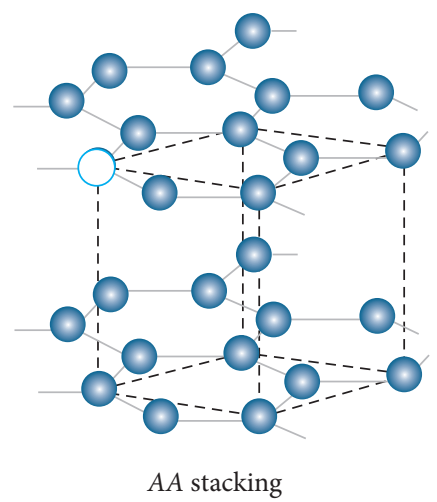

(a)

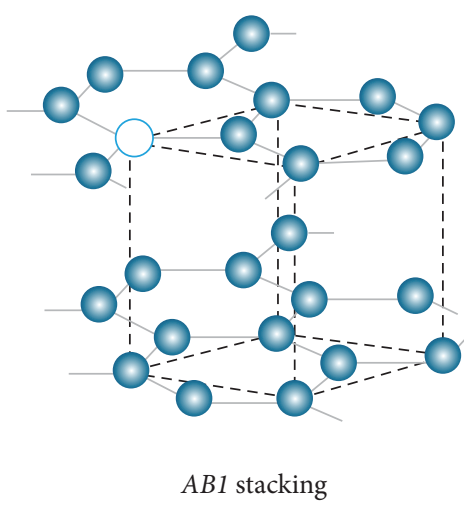

(b)

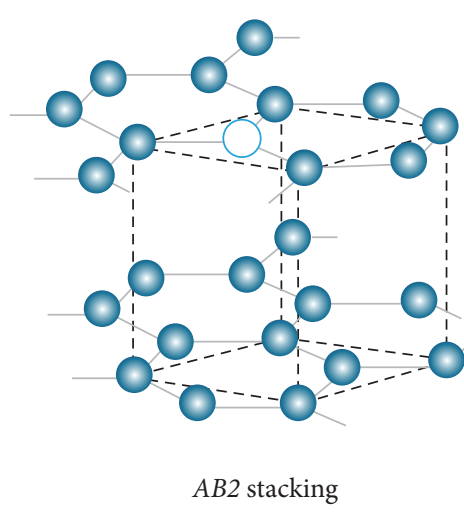

(c)

Figure 2: Schematic view of bilayer graphene with $A A$ and $A B$ stacking. The dotted line denotes the two-dimensional unit cell. The open circles denote the substitution sites of dopants. For $A B 1$ and $A B 2$ stacking patterns, see text. Reproduced with permission from [57], copyright 2015, Elsevier.

We further study the relative stabilities of $\mathrm{B}$ - and $\mathrm{N}$-doped bilayer graphenes among three types of $A A, A B 1$, and $A B 2$ stacking patterns. Table 1 shows the formation energy differences between $A A$ - and $A B 1$-, $A B 2$-stacked bilayer graphenes doped with $\mathrm{B}$ and $\mathrm{N}$ atoms. For the $\mathrm{B}$ - doped case, the formation energies of $A B$-stacked bilayer graphene for two kinds of doping site ( $A B 1$ and $A B 2)$ take similar values with only $6 \mathrm{meV}$ difference. For the $\mathrm{N}$-doped case, the formation energy of the $A B 1$-stacked graphene is considerably larger by $28 \mathrm{meV}$ than that of $A B 2$ stacking. This means that the substitution with $\mathrm{N}$ atom on top of the center of a hexagon is favored energetically rather than that on top of $\mathrm{C}$ atom. Thus, the formation energy for the substitution with $\mathrm{N}$ atom depends on the substitution site, whereas that for $\mathrm{B}$ atom does not depend on it. Furthermore, the $\mathrm{B}$ and $\mathrm{N}$ doping into bilayer graphene become more preferable in energy than those into monolayer graphene without depending on the stacking patterns.

2.2. Nitrogen-Vacancy Complex Defects in Graphene. We here investigate the atomic configurations and the energetics of nitrogen and atomic vacancy complexes, pyridine-type defects, in monolayer graphene (see Figures 3(b)-3(e)). When the nitrogen is doped into graphene, not only the substitutional $\mathrm{N}$ defect but also the pyridine-type defects have been observed experimentally by X-ray photoelectron spectroscopy (XPS) measurements [24, 42]. However, it is difficult to identify the possible atomic configurations among the various formations of the pyridine-type defects.

We now consider five atomic configurations for $\mathrm{N}$ doped graphene as shown in Figures 3(a)-3(e) in order to discuss the energetics of nitrogen doping into graphene. In Figure 3(a), the substitutionally $\mathrm{N}$ defective graphene $\left(\mathrm{C}_{31} \mathrm{~N}\right)$ is depicted, where one $\mathrm{C}$ atom is substituted with $\mathrm{N}$ atom as already discussed. As pyridine-type configurations around a monovacancy, the trimerized $\left(\mathrm{C}_{28} \mathrm{~N}_{3}\right)$, the monomeric $\left(\mathrm{C}_{30} \mathrm{~N}\right)$, and the dimerized $\left(\mathrm{C}_{29} \mathrm{~N}_{2}\right)$ formations are shown in Figures 3(b)-3(d), respectively. In Figure 3(e), the tetramerized pyridine-type configuration in graphene
TABLE 1: Calculated formation energy difference between $A A$ - and $A B 1-(A B 2-)$ stacked bilayer graphenes doped with $\mathrm{B}$ and $\mathrm{N}$ atoms. Reproduced with permission from [57], copyright 2015 Elsevier.

\begin{tabular}{lccc}
\hline & \multicolumn{2}{c}{ Formation energy difference $(\mathrm{meV})$} & \\
& $A A$ & $A B 1$ & $A B 2$ \\
\hline B-doped & 0 & 17 & 11 \\
N-doped & 0 & 44 & 16 \\
\hline
\end{tabular}

$\left(\mathrm{C}_{26} \mathrm{~N}_{4}\right)$ is also illustrated, where four $\mathrm{N}$ atoms are arranged around a divacancy.

Before discussing the atomic structure of the pyridinetype defects around a monovacancy, we consider the structural properties of the undoped graphene with a monovacancy. In this case, the atomic configuration around the vacancy is relaxed under a Jahn-Teller distortion and the symmetry around the vacancy is lowered from $D_{3 h}$ to $C_{s}$. Thereby, two of three $\mathrm{C}$ atoms around the vacancy move closer together and a pentagon-like ring is formed (Figure 3(f)). However, the distance of these two $\mathrm{C}$ atoms is relatively long compared with the $\mathrm{C}-\mathrm{C}$ bond length of pristine graphene. This implies that the interaction between two $C$ atoms could be relatively weak. On the other hand, for the trimerized pyridine-type defects where one $\mathrm{C}$ atom is removed, three $\mathrm{N}$ atoms around the vacancy move away from one another, keeping $D_{3 h}$ symmetry (Figure $3(\mathrm{~b})$ ). The $\mathrm{N}$ atom is twofold coordinated with the resultant $\mathrm{C}$-N bond length of $1.33 \AA$, which is considerably shorter than the C-C bond length of pristine graphene sheet, $1.41 \AA$. We also consider monomeric and dimerized pyridine-type formations around the monovacancy. For the monomeric formation (Figure 3(c)), two C atoms around the monovacancy also approach each other. As a result, the covalent bond is formed between two $\mathrm{C}$ atoms. This behavior is similar to that around the monovacancy in undoped graphene, as discussed above. In the case of the dimerized pyridine-type defects (Figure 3(d)), the $\mathrm{C}$ atom around the monovacancy has one $s p^{2}$-dangling bond since the distance between the $\mathrm{C}$ atom and the $\mathrm{N}$ atom around 


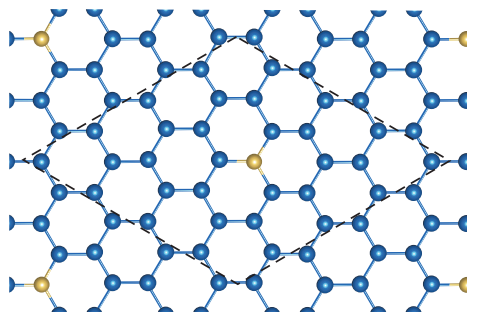

- $\mathrm{C}$ atom

- $\mathrm{N}$ atom

(a)

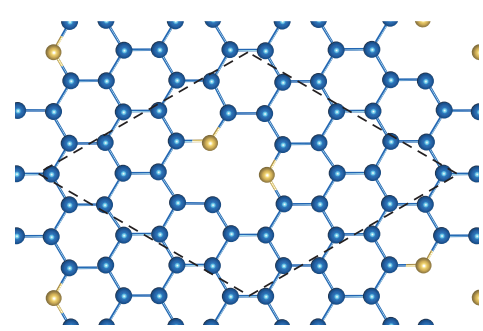

- $\mathrm{C}$ atom

- $\mathrm{N}$ atom

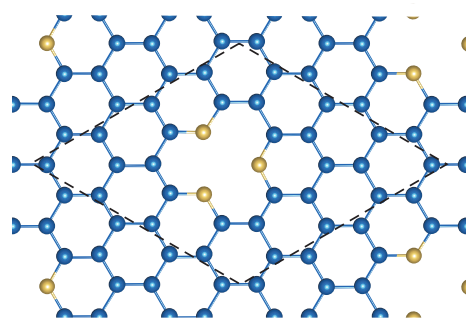

- $\mathrm{C}$ atom

- $\mathrm{N}$ atom

(b)

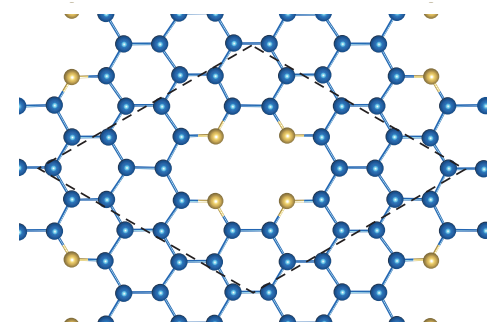

- $\mathrm{C}$ atom

- $\mathrm{N}$ atom

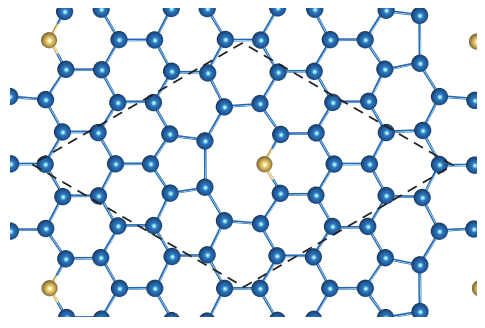

- $\mathrm{C}$ atom

- $\mathrm{N}$ atom

(c)

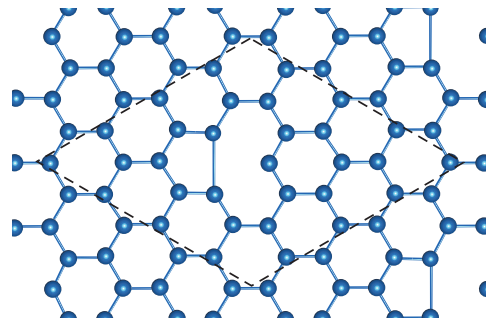

- $\mathrm{C}$ atom

- $\mathrm{N}$ atom

(f)

(d)

(e)

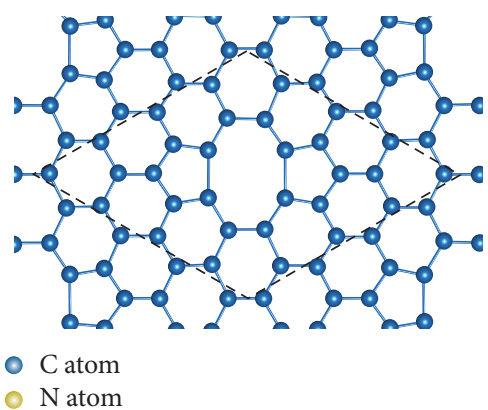

(g)

Figure 3: Atomic configurations of various $\mathrm{N}$ defects and undoped defective graphene. The dotted line denotes the unit cell. Atomic configurations of $\mathrm{N}$-doped graphene and undoped defective graphene: (a) substitutional nitrogen defect $\left(\mathrm{C}_{31} \mathrm{~N}\right)$, (b) trimerized pyridinetype defect $\left(\mathrm{C}_{28} \mathrm{~N}_{3}\right)$, (c) monomeric pyridine-type defect $\left(\mathrm{C}_{30} \mathrm{~N}\right)$, (d) dimerized pyridine-type defect $\left(\mathrm{C}_{29} \mathrm{~N}_{2}\right)$, (e) tetramerized pyridine-type defect $\left(\mathrm{C}_{26} \mathrm{~N}_{4}\right)$, (f) a monovacancy $\left(\mathrm{C}_{31}\right)$, and $(\mathrm{g})$ a divacancy $\left(\mathrm{C}_{30}\right)$. In each case, the geometry is fully optimized in the framework of the density-functional theory. The dashed lines show the supercell. Reproduced with permission from [47], copyright 2011, the American Physical Society.

the monovacancy is large, which is approximately $2.46 \AA$ [ $47-$ 49]. This structural behavior is quite different from that of $\mathrm{N}$ doped $(10,0)$ carbon nanotubes since the $\mathrm{C}-\mathrm{N}$ bond around the vacancy is formed [50]. These differences may be caused from curvature effects in carbon nanotubes [48].

We also study the tetramerized pyridine-type defects around the divacancy where two $\mathrm{C}$ atoms are removed (Figure 3(e)). The optimized C-N bond lengths are within 1.32-1.33 $\AA$ and the interatomic distances among four $\mathrm{N}$ atoms remain so long that $\mathrm{N}$ atoms keep away from one another as in the case of trimerized pyridine-type defects. On the other hand, for the undoped graphene with the divacancy, the neighboring $\mathrm{C}$ atoms around the divacancy move close, and the pentagon-octagon-pentagon rings are formed after the atomic configurations around the divacancy are relaxed (Figure 3(g)). Thus, $\mathrm{C}$ atoms around the divacancy prefer the configuration of the C-C bond to saturate dangling bonds, whereas forming the N-N bond around the vacancy seems to be unfavorable since $\mathrm{N}$ atoms around the vacancy should possess the lone-pair electrons.

We move on to discussions on the stabilities of various $\mathrm{N}$-doped graphenes. To discuss the energetics of various $\mathrm{N}$ doped graphenes, we also calculate the formation energies $E_{f}$ defined by (1). Table 2 shows the calculated formation energies of various $\mathrm{N}$-defect configurations. The trimerized pyridine-type formation has the lowest formation energy $\left(E_{f}=2.51 \mathrm{eV}\right)$ among various pyridine-type defect configurations. On the other hand, the formation energy of 
TABLE 2: Calculated formation energies of various pyridine-type defective graphenes.

\begin{tabular}{lccc}
\hline \multicolumn{4}{c}{ Formation energy $(\mathrm{eV})$} \\
$E_{f}\left(\mathrm{C}_{28} \mathrm{~N}_{3}\right)$ & $E_{f}\left(\mathrm{C}_{29} \mathrm{~N}_{2}\right)$ & $E_{f}\left(\mathrm{C}_{30} \mathrm{~N}\right)$ & $E_{f}\left(\mathrm{C}_{31} \mathrm{~N}\right)$ \\
\hline 2.51 & 2.55 & 5.61 & 4.28 \\
\hline
\end{tabular}

the tetramerized pyridine-type formation is $2.58 \mathrm{eV}$, which is larger by only $0.04 \mathrm{eV}$ compared with that of the trimerized one. Thus, this small difference infers that both defects are possible formations, and they are candidates for pyridinetype configurations in graphene. As shown in the previous subsection, the formation energy of the substitutional $\mathrm{N}$ doped graphene is $0.32 \mathrm{eV}$ and it is the lowest among the various $\mathrm{N}$-defect configurations, which suggests that the substitutional N-defect formation is the most plausible when nitrogen is doped into monolayer graphene.

We further extend discussions on energetics of $\mathrm{N}$ doped graphenes [47]. The formation energy discussed above implies the energetic preference regarding the doping with nitrogen into the perfect sheet of graphene. On the other hand, to address how the pyridine-type configurations are formed, the relative energies are defined by (2)-(5) which are comparing the total energy of the trimerized pyridinetype configurations directly with that of the substitutional $\mathrm{N}$ configuration. The relative energies are defined by

$$
\begin{aligned}
& E_{1}= E\left(\mathrm{C}_{28} \mathrm{~N}_{3}\right)+E\left(\mathrm{C}_{32}\right) \times 3 \\
&-\left[E\left(\mathrm{C}_{31} \mathrm{~N}\right) \times 3+E\left(\mathrm{C}_{31}\right)\right], \\
& E_{2}=E\left(\mathrm{C}_{28} \mathrm{~N}_{3}\right)+E\left(\mathrm{C}_{32}\right) \times 3 \\
&-\left[E\left(\mathrm{C}_{31} \mathrm{~N}\right) \times 3+E\left(\mathrm{C}_{30}\right)+E\left(\mathrm{C}_{1}\right)\right], \\
& E_{3}=E\left(\mathrm{C}_{28} \mathrm{~N}_{3}\right)+E\left(\mathrm{C}_{32}\right) \times 2 \\
&-\left[E\left(\mathrm{C}_{31} \mathrm{~N}\right) \times 2+E\left(\mathrm{C}_{30} \mathrm{~N}\right)\right], \\
& E_{4}=E\left(\mathrm{C}_{28} \mathrm{~N}_{3}\right)+E\left(\mathrm{C}_{32}\right)-\left[E\left(\mathrm{C}_{31} \mathrm{~N}\right)+E\left(\mathrm{C}_{29} \mathrm{~N}_{2}\right)\right] .
\end{aligned}
$$

Here, $E\left(\mathrm{C}_{32}\right), E\left(\mathrm{C}_{31}\right)$, and $E\left(\mathrm{C}_{30}\right)$ are the total energies of pristine graphene, undoped graphene with a monovacancy, and undoped graphene with a divacancy, respectively, and $E\left(\mathrm{C}_{28} \mathrm{~N}_{3}\right), E\left(\mathrm{C}_{29} \mathrm{~N}_{2}\right), E\left(\mathrm{C}_{30} \mathrm{~N}\right)$, and $E\left(\mathrm{C}_{31} \mathrm{~N}\right)$ are total energies of $\mathrm{N}$-doped graphenes with trimerized, dimerized, monomeric pyridine-type defects, and substitutional $\mathrm{N}$ doped defect, respectively. $E\left(\mathrm{C}_{1}\right)$ is energy per atom in a pristine graphene which is the same as the chemical potential $\mu_{\mathrm{C}}$ defined above.

The relative energies of $E_{1}$ and $E_{2}$ indicate the energetic preferences when there exist the monovacancy and the divacancy in graphene [51], whereas the relative energies of $E_{3}$ and $E_{4}$ discuss the energetic preferences when the monomeric and the dimerized pyridine-type defects are present, respectively. Table 3 shows calculated relative energies of $\mathrm{N}$-doped graphenes. The trimerized pyridinetype configuration becomes energetically favorable by $E_{1}=$ $-6.76 \mathrm{eV}$ rather than the substitutional nitrogen configuration in the presence of the monovacancy defect in graphene.
TABLE 3: Calculated relative energies of $\mathrm{N}$-doped graphenes. The relative energies $E_{1}-E_{4}$ are defined in (2)-(5), respectively.

\begin{tabular}{lccc}
\hline \multicolumn{4}{c}{ Relative energy $(\mathrm{eV})$} \\
$E_{1}$ & $E_{2}$ & $E_{3}$ & $E_{4}$ \\
\hline-6.76 & -7.13 & -3.75 & -2.10 \\
\hline
\end{tabular}

When the divacancy is present in graphene, the trimerized pyridine-type configuration is also favored by $E_{2}=-7.13 \mathrm{eV}$ in energy. We next consider the relative energies $E_{3}$ and $E_{4}$ defined by (4) and (5), corresponding to the growth processes of the monomeric and the dimerized pyridinetype defects into the trimerized pyridine-type defects. The $E_{3}$ value of $-3.75 \mathrm{eV}$ means that the monomeric configuration is less stable than the trimerized one by $3.75 \mathrm{eV}$ if two substitutional nitrogen defects are also present and can react with the monomeric pyridine-type defect. In the case of relative energy $E_{4}=-2.10 \mathrm{eV}$, the dimerized nitrogen formation is also less stable than the trimerized one by $2.10 \mathrm{eV}$. We thus find that the trimerized pyridine-type formation is more preferable in energy than the substitutional nitrogendefect formation when there is the vacancy in graphene. Furthermore, the trimerized formation in the pyridine-type defect is found to be energetically preferred compared with the monomeric and the dimerized pyridine-type formations. These results of relative energies indicate that the trimerized pyridine-type formation is formed from the substitutional $\mathrm{N}$ defect by way of the monomeric and dimerized formations.

\section{Electronic Structures}

In this section, the electronic properties of $\mathrm{B}$ and $\mathrm{N}$ defects in monolayer and bilayer graphenes are presented. In Section 3.1, the energy bands of B-doped and N-doped graphenes are exhibited, and the spatial distributions of the electron densities induced by $\mathrm{B}$ and $\mathrm{N}$ defects are also shown. In Section 3.2, the scanning tunneling microscopy (STM) images of $\mathrm{B}$-doped and $\mathrm{N}$-doped graphenes are demonstrated. The work functions of $\mathrm{B}\left(\mathrm{N}_{-}\right)$doped as well as undoped graphene are shown and the polarity variation induced by the $\mathrm{B}$ and $\mathrm{N}$ defects is discussed in Section 3.3.

3.1. Energy-Band Structures. We begin with introducing the energy-band structure of the pristine monolayer graphene, as shown in Figure 4(a). The linear dispersions are seen near the Fermi energy and they cross at the Dirac point. This means that the electrons and holes behave as massless Dirac fermions and they can move with the speed of 300 times less than the speed of light. Moreover, graphene becomes a zerogap material [4].

We next show the energy-band structures of substitutional $\mathrm{B}$ and $\mathrm{N}$ defects in monolayer graphene in Figures 4(b) and 4(c), respectively. For the B-doped case, the acceptor-like state related to the B defect appears near the Fermi energy, whereas in the case of substitutionally $\mathrm{N}$-doped graphene, the donor-like state associated with the N-impurity defect is observed near the Fermi energy. The substitutionally Bdoped and $\mathrm{N}$-doped graphenes thus may possess $p$-type and 


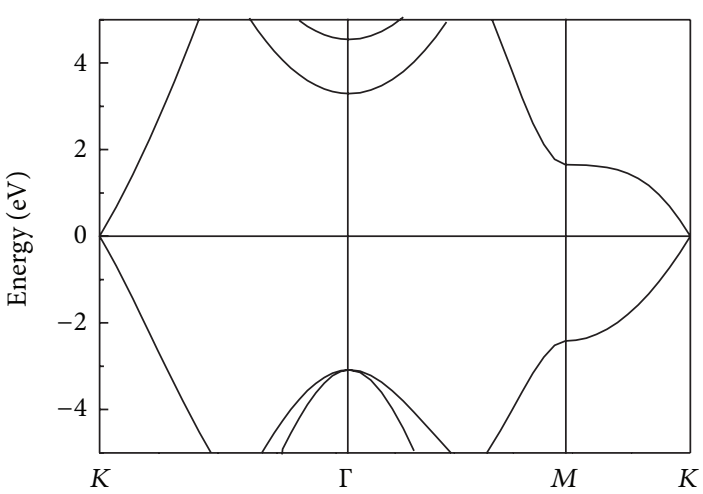

(a)

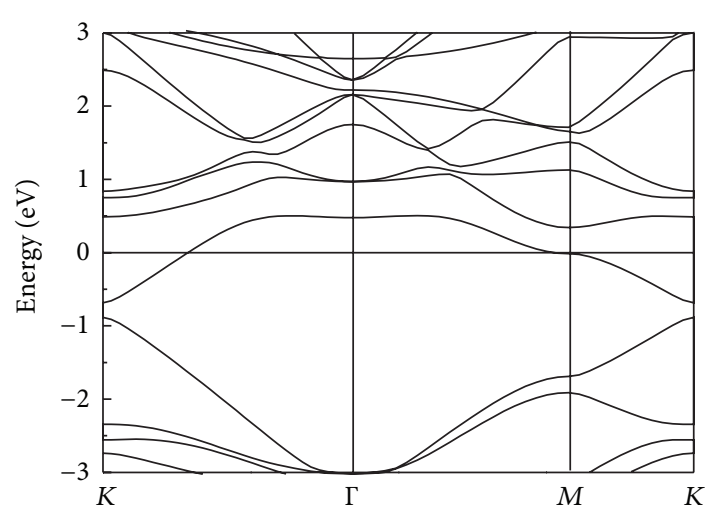

(c)

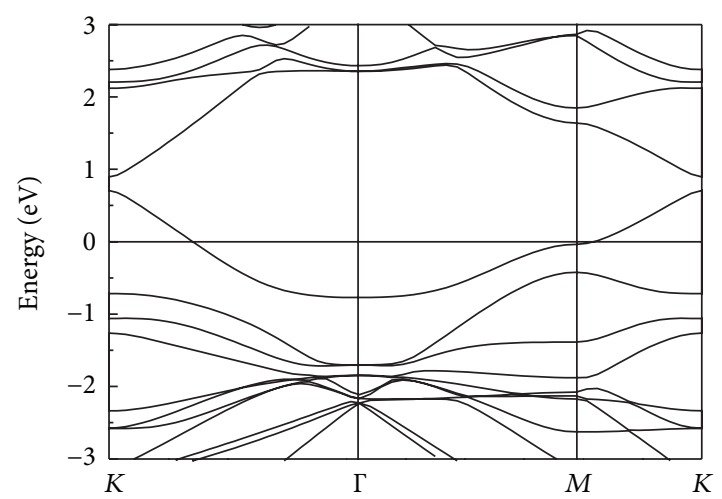

(b)

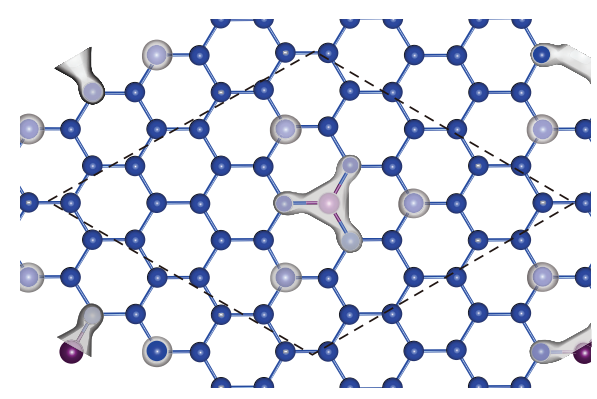

- $\mathrm{C}$ atom

- B atom

- $\mathrm{N}$ atom

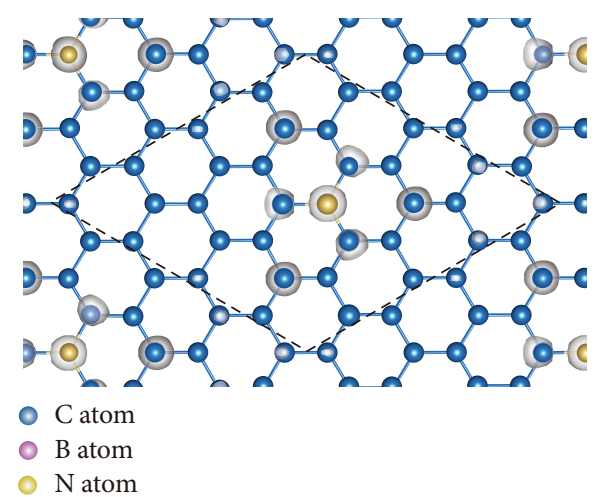

(e)

Figure 4: Energy-band structures of (a) pristine, (b) B-doped, (c) N-doped graphenes, and the isosurfaces of electron-state densities related to (d) $\mathrm{B}$ and (e) $\mathrm{N}$ defects in monolayer graphene. The isosurface value of electron density is set to 0.03 electron $/ \AA^{3}$. The Fermi energy is set to be zero. The energy bands of the pristine graphene are plotted using $1 \times 1$ unit cell, whereas those of $\mathrm{B}(\mathrm{N}-)$ doped graphene are plotted using $4 \times 4$ supercell.

$n$-type conducting properties since $\mathrm{B}$ and $\mathrm{N}$ atoms have deficit and extra one electrons compared with $\mathrm{C}$ atom, respectively. In Figures 4(d) and 4(e), the isosurfaces of electron densities of substitutionally B-doped and $\mathrm{N}$-doped graphenes at $\Gamma$ point are displayed, respectively. The electron densities of boron and nitrogen impurities are delocalized around the $\mathrm{B}$ atom and the $\mathrm{N}$ atom, respectively. It is interesting that there are differences between $\mathrm{B}$ and $\mathrm{N}$ defects in the spatial distributions of electron densities: the electron density of the $\mathrm{B}$ defect is extended to three $\mathrm{C}$ atoms around the $\mathrm{B}$ atom, which consists of a triangle-like shape, whereas that of the $\mathrm{N}$ defect is distributed above $\mathrm{N}$ atom and three $\mathrm{C}$ atoms, which appears to be individual four spots. In addition, this delocalization in the electron density is also observed in other defects with threefold symmetry such as impurity doped and impurity adsorbed graphenes [52, 53]. 


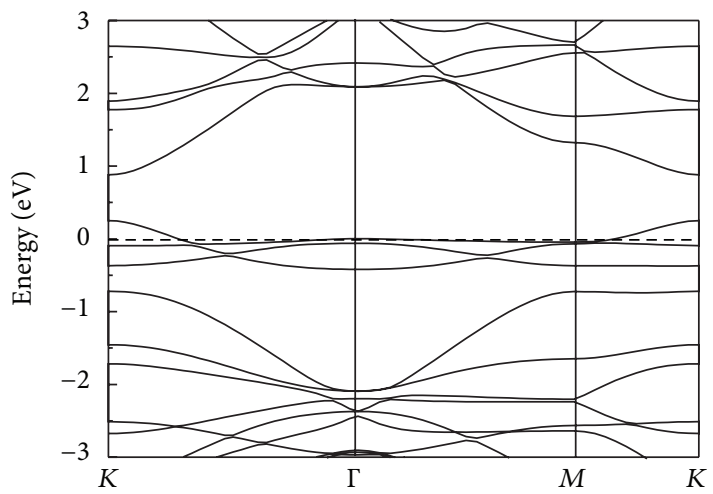

(a)

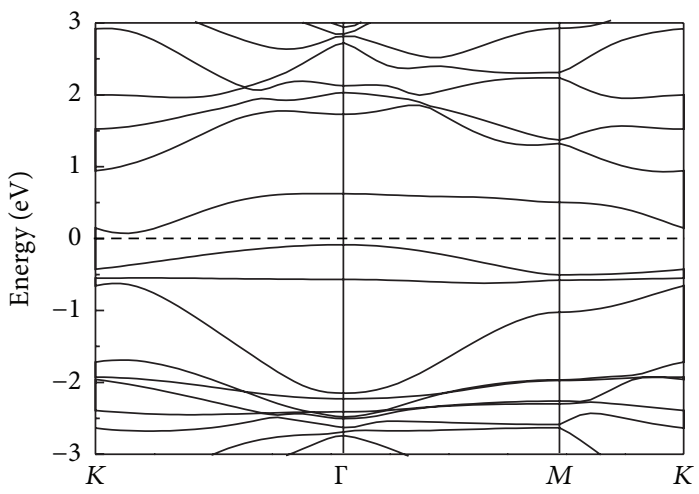

(c)

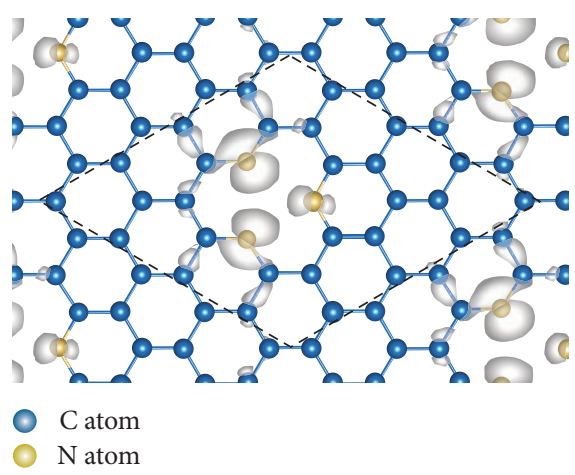

(e)

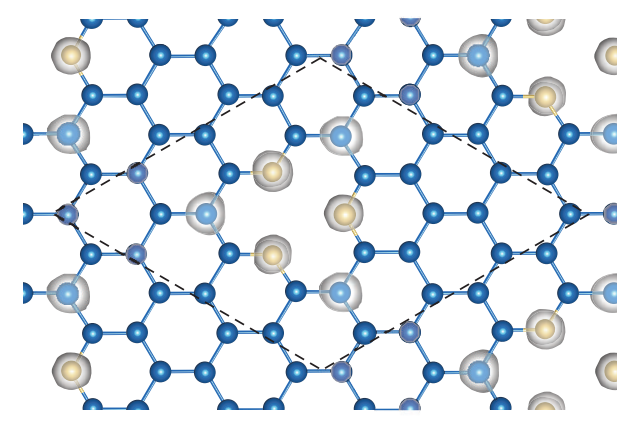

- $\mathrm{C}$ atom

N atom

(b)

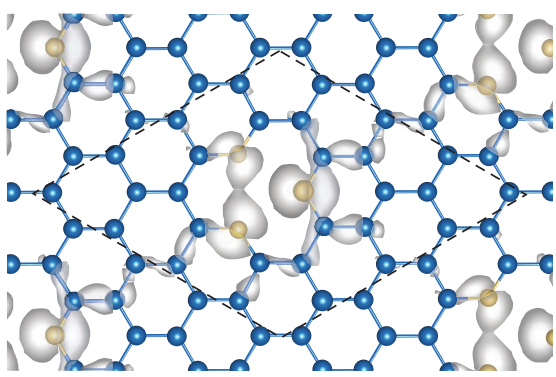

(d)

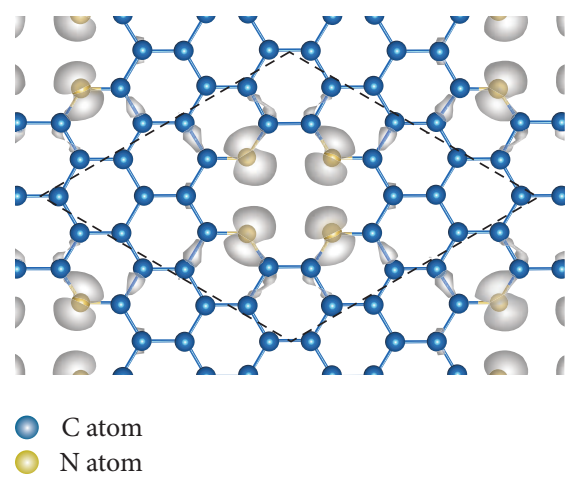

(f)

Figure 5: (a) Energy band and (b), (d)-(e) isosurfaces of electron densities of the three states associated with the trimerized pyridine-type defects in graphene (see text). (c) Energy band and (f) isosurface of electron density of tetramerized pyridine-type defects in graphene. The isosurface value of electron density is set to 0.03 electrons $/ \AA^{3}$. The Fermi energy is set to zero. The dashed lines show the supercell. Reproduced with permission from [47], copyright 2011, the American Physical Society.

The energy bands of graphene with trimerized pyridinetype defects are shown in Figure 5(a). Being different from the substitutional nitrogen defective graphene, the impurity states induced by trimerized pyridine-type defects are located within the valence band of graphene. This is because the number of electrons of trimerized pyridine-type defective graphene is deficient compared with a pristine graphene. The electron deficiency induces the acceptor-type state and the trimerized pyridine-type graphene exhibits $p$-type doping property. In addition, it is seen that there are three impurity states related to the trimerized pyridine-type defects near the Fermi level. Figures 5(b) and 5(d)-5(e) exhibit the isosurfaces of electronic states of a single state and doubly degenerated states at $\Gamma$ point, which are composed of one $p_{z}$-orbital and two $p_{x y}$-orbital shapes, respectively. Interestingly, the feature of impurity states of trimerized pyridine-type defect is similar to that of unrelaxed structure of undoped graphene with the monovacancy [54]. 
Figure 5(c) shows the energy band of the tetramerized pyridine-type defective graphene. It is observed that there are several N-related impurity states near the Fermi level, as in the case of the trimerized pyridine-type defects in graphene, and one of them is unoccupied electronic state. Figure 5(f) illustrates the isosurface of electron density above the valence-band maximum at $\Gamma$ point. The spatial distributions of the impurity state are localized at four $\mathrm{N}$ atoms around the divacancy and it forms $p$-like orbital shapes localized at $\mathrm{N}$ atoms.

We here examine the electronic structures of bilayer graphene. Figure 6 shows the energy-band structures of pristine, B-doped and $\mathrm{N}$-doped bilayer graphenes. The energyband structure of the pristine $A A$-stacked graphene is quite different from that of the pristine $A B$-stacked graphene (Figures 6(a) and 6(d)). For the $A A$ stacking, the linear dispersions appear near the Fermi level as in the case of the monolayer graphene. The linear dispersion of the $A A$-stacked graphene is experimentally observed [55]. On the other hand, for the $A B$ stacking, the parabolic bands are seen near the Fermi level due to asymmetric interactions between upper and lower layers in graphene bilayer [56]. In addition, both of $A A$ - and $A B$-stacked graphenes are still gapless materials.

Irrespectively of the stacking patterns, the substitutional $\mathrm{B}$ and $\mathrm{N}$ defects give rise to the acceptor-like and the donor-like states near the Fermi energy, respectively, and the charge carriers are induced on both two layers of bilayer graphene [57]. The band gaps appear due to the interactions of dopants arising from lateral periodicity. The band gaps are $0.11 \mathrm{eV}, 0.17 \mathrm{eV}$, and $0.32 \mathrm{eV}$ for B-doped $A A-, A B 1$-, and $A B 2$-stacked graphenes, respectively, and they are $0.09 \mathrm{eV}$, $0.13 \mathrm{eV}$, and $0.30 \mathrm{eV}$ for $\mathrm{N}$-doped $A A-, A B 1$-, and $A B 2$-stacked graphenes, respectively. It is interesting that the band gaps vary depending on the stacking patterns, and in addition the band gaps of B-doped systems are somewhat larger than those of $\mathrm{N}$-doped ones. The feature of the band gaps is in agreement with that in a previous report [58-60]. The energy dispersions near the Fermi energy of $A A$-stacked graphenes are similar to those of $A B 1$ - and $A B 2$-stacked graphenes for both B-doped and $\mathrm{N}$-doped cases, leading to similar STM images as will be discussed later.

3.2. Scanning Tunneling Microscopy Images. Scanning tunneling microscopy (STM) measurement is an effective method to observe the local electronic structures near lattice defects such as atomic vacancies and impurities in atomic level. We here demonstrate the STM images of B and N defects in monolayer and bilayer graphenes. The STM images of various defects in graphene sheets are generated within the Tersoff-Hamann (TH) approach $[61,62]$. Due to its simplicity, this approach is well known to be valid for many systems [63-66]. In this approach, the tunneling current $I$ is assumed to be proportional to the local density of states (LDOS) of the surface at the tip position integrated over an energy range restricted by the applied bias voltage $[61,62,67]$. Consequently, the STM images can be constructed from the isosurface of the spatial distribution integrated by the LDOS $\rho(\mathbf{r}, \varepsilon)$ at spatial points $\mathbf{r}=(x, y, z)$ and energy $\varepsilon$ by several sampling $k$ points of the Brillouin zone over the energy range from the Fermi energy $E_{F}$ to $E_{F}+\mathrm{eV}$ with applied voltage $V$; that is,

$$
I \sim \int_{E_{F}}^{E_{F}+\mathrm{eV}} \rho(\mathbf{r}, \varepsilon) d \varepsilon
$$

We now examine the STM images of several N-impurity defects in monolayer graphene [47]. Figure 7(a) shows the STM image around substitutional N-defect in graphene at a bias voltage of $+0.5 \mathrm{eV}$ (conduction band). It is observed that there are three bright protrusions at three $\mathrm{C}$ atoms around the $\mathrm{N}$ atom [68]. The dark area in the STM image is seen above the $\mathrm{N}$ atom as if the $\mathrm{N}$ atom were absent although the spatial distribution of the impurity-induced state is also extended above the $\mathrm{N}$ atom (see Figure 4(e)). The difference is caused from how the spatial distributions of LDOS protrude above the $\mathrm{N}$ atom and the surrounding three $\mathrm{C}$ atoms: the LDOS above three $\mathrm{C}$ atoms are more delocalized compared with that above the $\mathrm{N}$ atom. Thereby, the STM image at three $\mathrm{C}$ atoms near the $\mathrm{N}$ atom has three bright spots, whereas the STM image at the $\mathrm{N}$ atom looks dark.

Figure 7(b) exhibits the STM image of the trimerized pyridine-type defects in graphene at a bias voltage of $-0.5 \mathrm{eV}$ (valence band). There exist three bright spots localized at three $\mathrm{N}$ atoms near the carbon vacancy, and it seems to form a triangular hillock since the spatial distribution of two Ndefect states near the Fermi level has $p$-orbital character protruding toward the carbon vacancy (Figures 5(d) and 5(e)). In addition, the STM image exhibits oscillations of electron density with $C_{3}$ symmetry propagating outwards from the center of the vacancy. Similar features are also observed in the case of the point defect in graphene [54]. Finally, we show in Figure 7(c) the STM image of tetramerized pyridinetype defects in graphene. In this case, the STM image has two large oval-shaped bright distributions consisting of four bright spots located at $\mathrm{N}$ atoms because the $\mathrm{N}$ atoms have the $p$-orbital shaped electron density protruding toward the carbon vacancy (Figure 5(f)). Similar oval-shaped protrusions rotated by $\pi / 2$ compared with those in Figure 7(c) have been reported, corresponding to the pentagon-octagon-pentagon structure in Figure 3(g) [54].

We also study the STM images of B-doped and Ndoped bilayer graphenes shown in Figure 8 [57]. The STM images of B-doped bilayer graphenes have a triangle-shaped bright area and show similar behavior irrespective of the stacking patterns. The triangle-shaped bright area in these simulated STM images is similar to that in the B-doped monolayer graphene sheet observed experimentally [15]. The STM images of the $\mathrm{N}$-doped graphene show sharp contrast to those of B-doped ones. The STM images of N-doped bilayer graphene have three bright spots at three $\mathrm{C}$ atoms around the $\mathrm{N}$ atom and the dark area is seen above the $\mathrm{N}$ atom as if $\mathrm{N}$ atom is absent. This variation of contrast in the STM image is also observed in the case of the substitutional $\mathrm{N}$ defect in monolayer graphene as shown in Figure 7(a). Moreover, the STM images are also similar to one another for the three stacking patterns as in the case of the B-doped bilayers. 


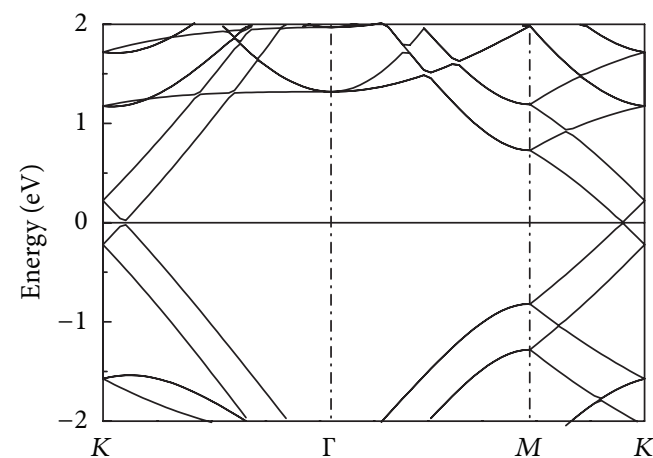

(a)

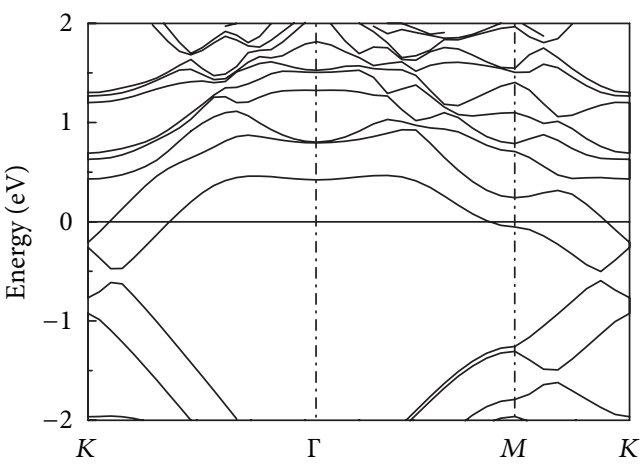

(c)

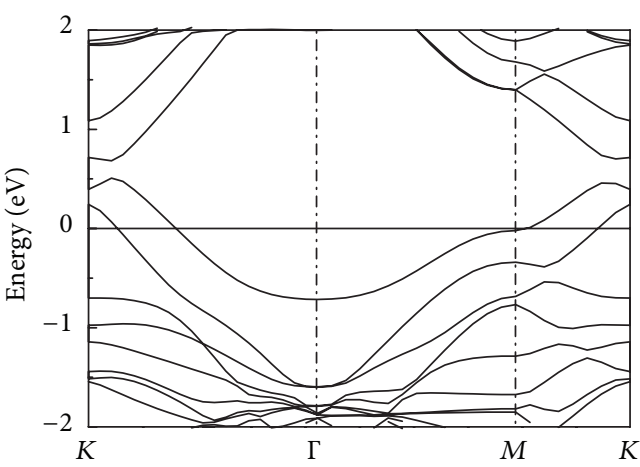

(e)

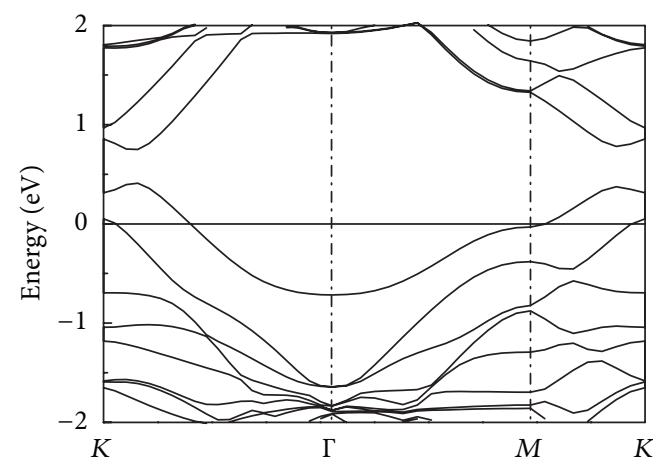

(g)

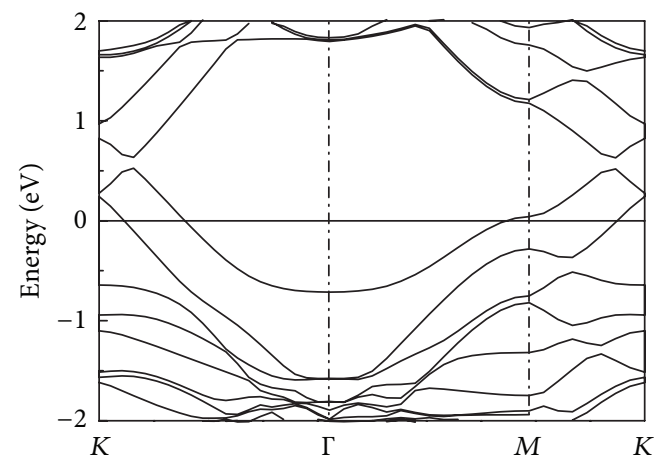

(b)

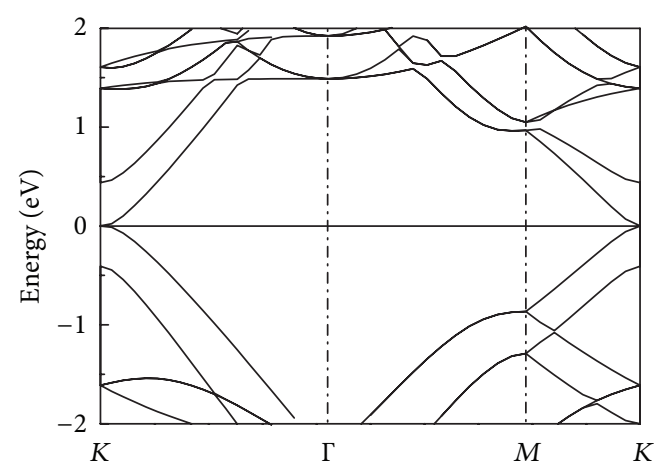

(d)

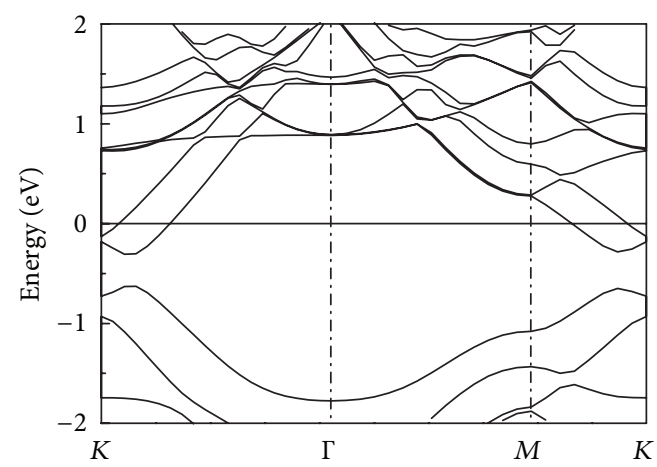

(f)

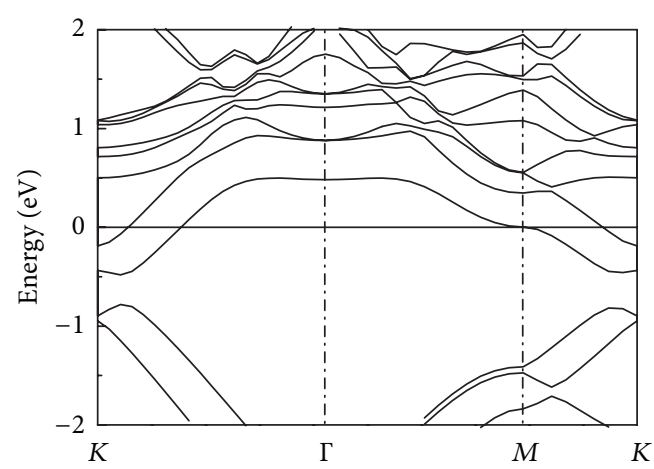

(h)

FIGURE 6: Electronic band structures of (a) undoped pristine $A A$-stacked, (b) B-doped $A A$-stacked, (c) N-doped $A A$-stacked, (d) undoped pristine $A B$-stacked, (e) B-doped $A B 1$-stacked, (f) N-doped $A B 1$-stacked, (g) B-doped $A B 2$-stacked, and (h) N-doped $A B 2$-stacked bilayer graphenes. The Fermi energy is set to be zero. Reproduced with permission from [57], copyright, 2015 Elsevier. 


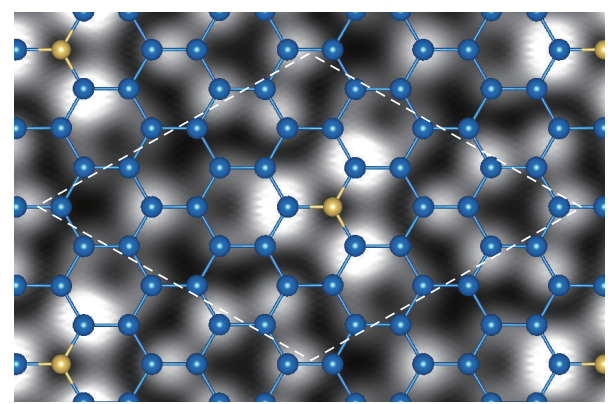

C atom

$\mathrm{N}$ atom

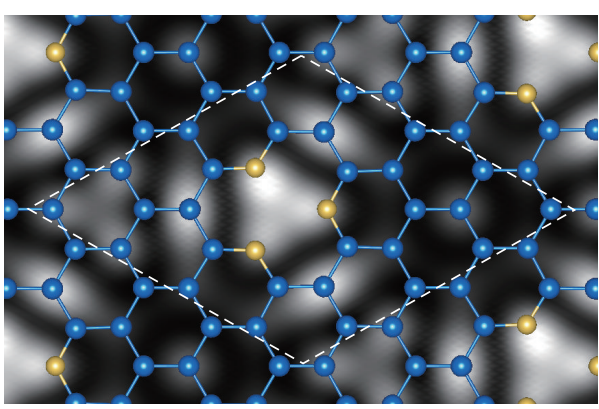

C atom

$\mathrm{N}$ atom

(a)

(b)

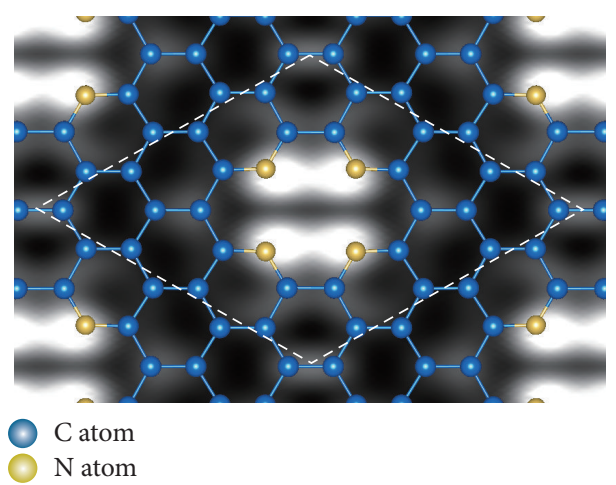

(c)

FIGURE 7: Simulated STM images of (a) substitutional nitrogen, (b) trimerized pyridine-type, and (c) tetramerized pyridine-type defects in graphene. The STM images are calculated at the applied bias voltages of (a) $+0.5 \mathrm{eV},(\mathrm{b})-0.5 \mathrm{eV}$, and (c) $-0.5 \mathrm{eV}$. The dotted line denotes the unit cell. Reproduced with permission from [47], copyright 2011, the American Physical Society.
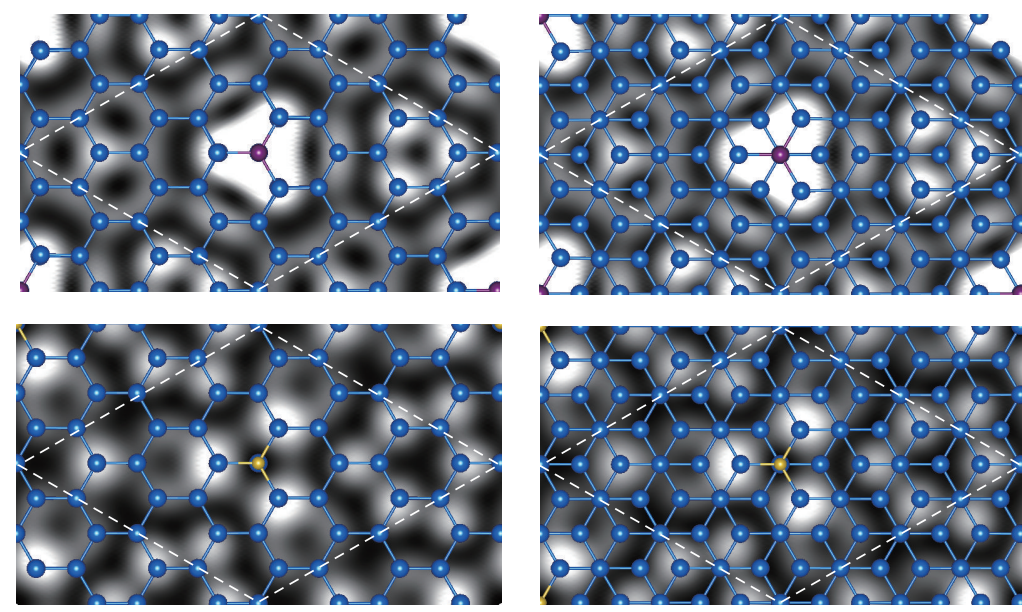

C atom
B atom
N atom

(a)
O C atom

B atom

- $\mathrm{N}$ atom

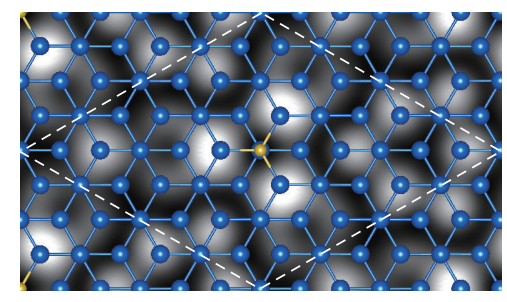

(b)
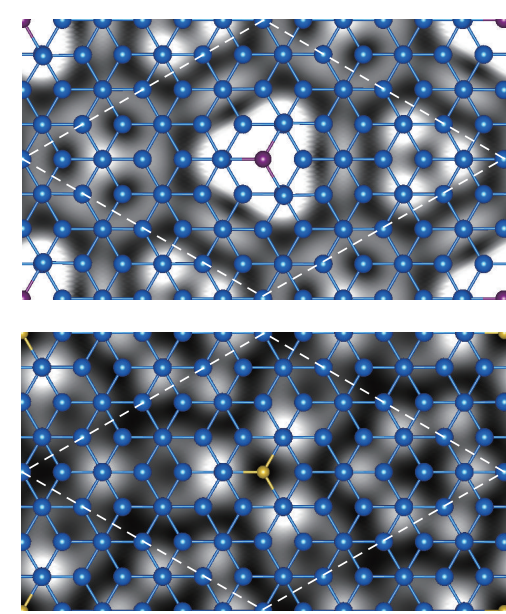

C atom

B atom

$\mathrm{N}$ atom

FIGURE 8: Simulated STM images of (a) $A A$-, (b) $A B 1$-, and (c) $A B 2$-stacked bilayer graphenes doped with $\mathrm{B}$ atom (upper panel) and N atom (lower panel). The STM images for B-doped and $\mathrm{N}$-doped cases are shown at the applied bias voltages of $+0.5 \mathrm{eV}$ and $-0.5 \mathrm{eV}$, respectively. Reproduced with permission from [57], copyright 2015, Elsevier. 


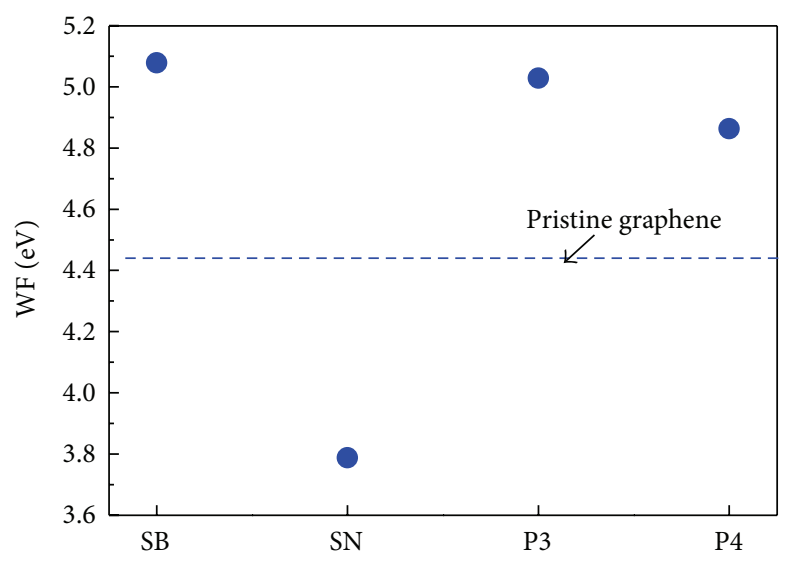

(a)

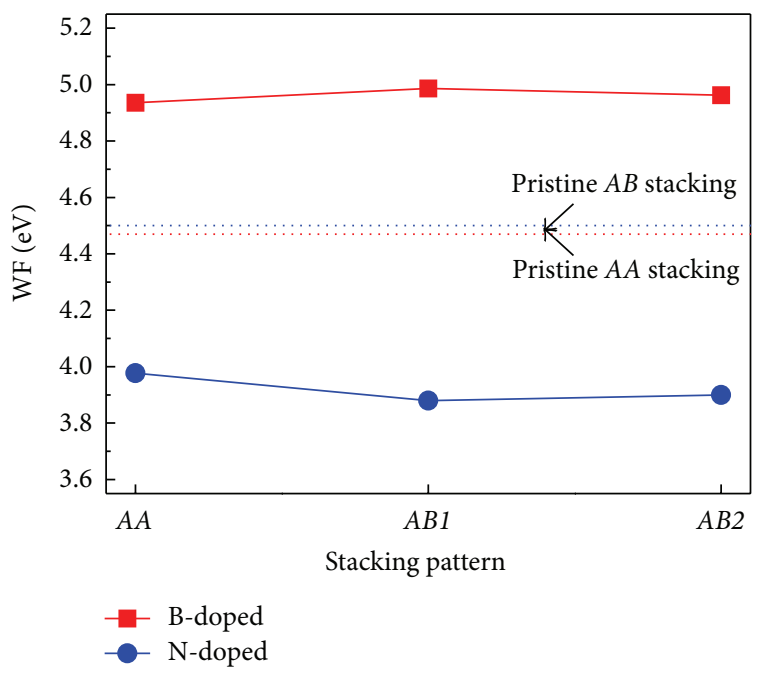

(b)

FIGURE 9: Work functions of pristine and $\mathrm{B}(\mathrm{N}-$ ) doped (a) monolayer with the substitutional $\mathrm{B}(\mathrm{SB})$, the substitutional $\mathrm{N}$ (SN), the trimerized pyridine-type (P3), and the tetramerized pyridine-type (P4) defects and (b) bilayer graphenes with $A A, A B 1$, and $A B 2$ stacking. The horizontal broken lines denote pristine monolayer and bilayer graphenes. (b) is taken from [57]. Reproduced with permission from [57], copyright 2015, Elsevier.

3.3. Work Functions. We finally study the work function $W$ of graphene monolayer and bilayer calculated by

$$
W=V_{\infty}-E_{F},
$$

where $V_{\infty}$ is vacuum level and $E_{F}$ is the Fermi energy. In Figure 9(a), the work functions of the pristine and (B)Ndoped monolayer graphenes are plotted. The calculated work function of the pristine monolayer graphene is $4.44 \mathrm{eV}$ [26], being in good agreement with the experimental value of $4.56 \mathrm{eV}$ [69]. When boron is doped substitutionally (SB), the work function increases, which reflects the $p$-type property, whereas in the case of the substitutional $\mathrm{N}(\mathrm{SN})$ defect the work function is reduced to $3.9 \mathrm{eV}$, being consistent with its $n$-type doping behavior. For both of the trimerized (P3) and the tetramerized pyridine-type (P4) defects, the work functions increase to approximately $5 \mathrm{eV}$ and the Fermi level moves away from the vacuum level compared with that of the pristine graphene, which means that the trimerized and the tetramerized pyridine-type defects induce the $p$-type property. Interestingly, the work function of impurity-doped graphene varies with depending on the concentration of the impurity defects [26].

We also study the work function of bilayer graphene. Figure 9(b) shows the work functions of the pristine, the Bdoped, and the $\mathrm{N}$-doped bilayer graphene. The calculated work functions of pristine bilayer graphenes with $A A$ and $A B$ stacking patterns are $4.47 \mathrm{eV}$ and $4.50 \mathrm{eV}$, respectively, which are somewhat larger than that of the pristine monolayer graphene [57]. It is in agreement with the experimentally observed work function of the pristine bilayer graphene [70, 71]. The work functions increase when $\mathrm{B}$ is doped for $A A$, $A B 1$, and $A B 2$ stacking patterns, whereas those of $\mathrm{N}$-doped bilayer graphene decrease without depending on the stacking patterns. Therefore, it is confirmed that B-doped bilayer graphenes show $p$-type doping properties, while $\mathrm{N}$-doped ones show $n$-type properties. In addition, it is interesting that, irrespective of the stacking patterns, the work functions of the B-doped bilayer graphene are relatively small than that of the B-doped monolayer graphene and those of the $\mathrm{N}$ doped bilayer graphene have larger value than that of the substitutionally $\mathrm{N}$-doped monolayer graphene, which infers that the charge carriers are induced on both of upper and lower layers in bilayer graphene.

\section{Conclusions}

Based on the first-principles total-energy calculations, the atomic structures, the energetics, and the electronic properties of boron and nitrogen defects in graphene monolayer and bilayer have been reviewed. The substitutional doping with $\mathrm{B}$ and $\mathrm{N}$ atoms into bilayer graphene becomes energetically favorable rather than that into monolayer graphene. The energetics corresponding to the growth processes from the substitutional $\mathrm{N}$ defect to the trimerized pyridine-type defects is clarified. The substitutional $\mathrm{B}$ and $\mathrm{N}$ defects give rise to the acceptor-like and the donor-like states in monolayer as well as bilayer graphene, respectively, and interestingly the pyridine-type defects induce the acceptor-like state. The simulated STM images of B and $\mathrm{N}$ defects exhibit distinctive STM images, which strongly depend on dopant types and the atomic configurations. The work functions increase and decrease when $\mathrm{B}$ and $\mathrm{N}$ impurities are doped into monolayer and bilayer graphenes.

\section{Disclosure}

Computations were done at Institute for Solid State Physics, the University of Tokyo, at Cybermedia Center, Osaka 
University, and at the Global Scientific Information and Computing Center, Tokyo Institute of Technology.

\section{Conflict of Interests}

The author declares that there is no conflict of interests regarding the publication of this paper.

\section{Acknowledgment}

This work was supported by MEXT Elements Strategy Initiative to Form Core Research Center through Tokodai Institute for Element Strategy and JSPS KAKENHI Grant no. 26390062.

\section{References}

[1] K. S. Novoselov, A. K. Geim, S. V. Morozov et al., "Electric field in atomically thin carbon films," Science, vol. 306, no. 5696, pp. 666-669, 2004.

[2] K. S. Novoselov, A. K. Geim, S. V. Morozov et al., "Twodimensional gas of massless Dirac fermions in graphene," Nature, vol. 438, no. 7065, pp. 197-200, 2005.

[3] S. V. Morozov, K. S. Novoselov, M. I. Katsnelson et al., "Giant intrinsic carrier mobilities in graphene and its bilayer," Physical Review Letters, vol. 100, no. 1, Article ID 016602, 2008.

[4] A. H. C. Neto, F. Guinea, N. M. R. Peres, K. S. Novoselov, and A. K. Geim, "The electronic properties of graphene," Reviews of Modern Physics, vol. 81, article 109, 2009.

[5] C. Lee, X. Wei, J. W. Kysar, and J. Hone, "Measurement of the elastic properties and intrinsic strength of monolayer graphene," Science, vol. 321, no. 5887, pp. 385-388, 2008.

[6] F. Guinea, M. I. Katsnelson, and A. K. Geim, "Energy gaps and a zero-field quantum hall effect in graphene by strain engineering," Nature Physics, vol. 6, no. 1, pp. 30-33, 2010.

[7] K. S. Novoselov, Z. Jiang, Y. Zhang et al., "Room-temperature quantum hall effect in graphene," Science, vol. 315, no. 5817, p. 1379, 2007.

[8] J. R. Williams, L. DiCarlo, and C. M. Marcus, "Quantum hall effect in a gate-controlled $p-n$ junction of graphene," Science, vol. 317, no. 5838, pp. 638-641, 2007.

[9] A. F. Young and P. Kim, "Quantum interference and Klein tunnelling in graphene heterojunctions," Nature Physics, vol. 5, no. 3, pp. 222-226, 2009.

[10] S. Iijima, "Helical microtubules of graphitic carbon," Nature, vol. 354, no. 6348, pp. 56-58, 1991.

[11] N. Hamada, S.-I. Sawada, and A. Oshiyama, "New onedimensional conductors: graphitic microtubules," Physical Review Letters, vol. 68, no. 10, article 1579, 1992.

[12] J.-C. Charlier, X. Blase, and S. Roche, "Electronic and transport properties of nanotubes," Reviews of Modern Physics, vol. 79, no. 2, pp. 677-732, 2007.

[13] S. Frank, P. Poncharal, Z. L. Wang, and W. A. de Heer, "Carbon nanotube quantum resistors," Science, vol. 280, no. 5370, pp. 1744-1746, 1998.

[14] Y.-B. Tang, L.-C. Yin, Y. Yang et al., "Tunable band gaps and $p$-type transport properties of boron-doped graphenes by controllable ion doping using reactive microwave plasma," ACS Nano, vol. 6, no. 3, pp. 1970-1978, 2012.
[15] L. Zhao, M. Levendorf, S. Goncher et al., "Local atomic and electronic structure of boron chemical doping in monolayer graphene," Nano Letters, vol. 13, no. 10, pp. 4659-4665, 2013.

[16] L. Yang, S. Jiang, Y. Zhao et al., "Boron-doped carbon nanotubes as metal-free electrocatalysts for the oxygen reduction reaction," Angewandte Chemie-International Edition, vol. 50, no. 31, pp. 7132-7135, 2011.

[17] R. Czerw, M. Terrones, J.-C. Charlier et al., "Identification of electron donor states in N-doped carbon nanotubes," Nano Letters, vol. 1, no. 9, pp. 457-460, 2001.

[18] D. Wei, Y. Liu, Y. Wang, H. Zhang, L. Huang, and G. Yu, "Synthesis of N-doped graphene by chemical vapor deposition and its electrical properties," Nano Letters, vol. 9, no. 5, pp. $1752-$ 1758, 2009.

[19] Y.-C. Lin, C.-Y. Lin, and P.-W. Chiu, "Controllable graphene Ndopeing with ammonia plasma," Applied Physics Letters, vol. 96, no. 13, Article ID 133110, 2010.

[20] P. Ayala, A. Grüneis, T. Gemming et al., "Tailoring N-doped single and double wall carbon nanotubes from a nondiluted carbon/nitrogen feedstock," Journal of Physical Chemistry C, vol. 111, no. 7, pp. 2879-2884, 2007.

[21] B. Guo, Q. Liu, E. Chen, H. Zhu, L. Fang, and J. R. Gong, "Controllable N-doping of graphene," Nano Letters, vol. 10, no. 12, pp. 4975-4980, 2010.

[22] Y.-S. Min, E. J. Bae, U. J. Kim et al., "Unusual transport characteristics of nitrogen-doped single-walled carbon nanotubes," Applied Physics Letters, vol. 93, no. 4, Article ID 043113, 2008.

[23] T. Susi, J. Kotakoski, R. Arenal et al., "Atomistic description of electron beam damage in nitrogen-doped graphene and singlewalled carbon nanotubes," ACS Nano, vol. 6, no. 10, pp. 8837$8846,2012$.

[24] T. Susi, T. Pichler, and P. Ayala, "X-ray photoelectron spectroscopy of graphitic carbon nanomaterials doped with heteroatoms," Beilstein Journal of Nanotechnology, vol. 6, no. 1, pp. 177-192, 2015.

[25] S. K. Hong and S. Jeong, "Nitrogen doping and chirality of carbon nanotubes," Physical Review B, vol. 70, no. 23, Article ID 233411, 4 pages, 2004.

[26] Y. Fujimoto and S. Saito, "Hydrogen adsorption and anomalous electronic properties of nitrogen-doped graphene," Journal of Applied Physics, vol. 115, no. 15, Article ID 153701, 2014.

[27] L. S. Panchakarla, K. S. Subrahmanyam, S. K. Saha et al., "Synthesis, structure, and properties of boron- and nitrogendoped graphene," Advanced Materials, vol. 21, no. 46, pp. 47264730, 2009.

[28] D. Usachov, O. Vilkov, A. Grüneis et al., "Nitrogen-doped graphene: efficient growth, structure, and electronic properties," Nano Letters, vol. 11, no. 12, pp. 5401-5407, 2011.

[29] M. Cattelan, S. Agnoli, M. Favaro et al., "Microscopic view on a chemical vapor deposition route to boron-doped graphene nanostructures," Chemistry of Materials, vol. 25, no. 9, pp. 14901495, 2013.

[30] J. Gebhardt, R. J. Koch, W. Zhao et al., "Growth and electronic structure of boron-doped graphene," Physical Review BCondensed Matter and Materials Physics, vol. 87, no. 15, Article ID 155437, 2013.

[31] S. Sandoval, N. Kumar, A. Sundaresan, C. N. R. Rao, A. Fuertes, and G. Tobias, "Enhanced thermal oxidation stability of reduced graphene oxide by nitrogen doping," Chemistry A European Journal, vol. 20, pp. 11999-12003, 2014. 
[32] H. M. Jeong, J. W. Lee, W. H. Shin et al., "Nitrogen-doped graphene for high-performance ultracapacitors and the importance of nitrogen-doped sites at basal planes," Nano Letters, vol. 11, no. 6, pp. 2472-2477, 2011

[33] K. Gopalakrishnan, A. Govindaraj, and C. N. R. Rao, "Extraordinary supercapacitor performance of heavily nitrogenated graphene oxide obtained by microwave synthesis," Journal of Materials Chemistry A, vol. 1, no. 26, pp. 7563-7565, 2013.

[34] G. Kim, S.-H. Jhi, and N. Park, "Effective metal dispersion in pyridinelike nitrogen doped graphenes for hydrogen storage," Applied Physics Letters, vol. 92, no. 1, Article ID 013106, 2008.

[35] Y. Wang, Y. Shao, D. W. Matson, J. Li, and Y. Lin, "Nitrogendoped graphene and its application in electrochemical biosensing," ACS Nano, vol. 4, no. 4, pp. 1790-1798, 2010.

[36] Y. J. Cho, H. S. Kim, H. Im et al., "Nitrogen-doped graphitic layers deposited on silicon nanowires for efficient lithium-ion battery anodes," Journal of Physical Chemistry C, vol. 115, no. 19, pp. 9451-9457, 2011.

[37] C. Berger, Z. Song, X. Li et al., "Electronic confinement and coherence in patterned epitaxial graphene," Science, vol. 312, no. 5777, pp. 1191-1196, 2006.

[38] Y. Zhang, Y.-W. Tan, H. L. Stormer, and P. Kim, "Experimental observation of the quantum Hall effect and Berry's phase in graphene," Nature, vol. 438, no. 7065, pp. 201-204, 2005.

[39] E. V. Castro, K. S. Novoselov, S. V. Morozov et al., "Biased bilayer graphene: semiconductor with a gap tunable by the electric field effect," Physical Review Letters, vol. 99, no. 21, Article ID 216802, 2007.

[40] L. Britnell, R. V. Gorbachev, R. Jalil et al., "Field-effect tunneling transistor based on vertical graphene heterostructures," Science, vol. 335, no. 6071, pp. 947-950, 2012.

[41] Y. Zhang, T.-T. Tang, C. Girit et al., "Direct observation of a widely tunable bandgap in bilayer graphene," Nature, vol. 459, no. 7248, pp. 820-823, 2009.

[42] C. N. R. Rao, K. Gopalakrishnan, and A. Govindaraj, "Synthesis, properties and applications of graphene doped with boron, nitrogen and other elements," Nano Today, vol. 9, no. 3, pp. 324343, 2014.

[43] C. N. R. Rao, A. K. Sood, K. S. Subrahmanyam, and A. Govindaraj, "Graphene: the new two-dimensional nanomaterial," Angewandte Chemie - International Edition, vol. 48, no. 42, pp. 7752-7777, 2009.

[44] Z. Liu, K. Suenaga, P. J. F. Harris, and S. Iijima, "Open and closed edges of graphene layers,” Physical Review Letters, vol. 102, no. 1, Article ID 015501, 2009.

[45] J. Lin, W. Fang, W. Zhou et al., "AC/AB stacking boundaries in bilayer graphene," Nano Letters, vol. 13, no. 7, pp. 3262-3268, 2013.

[46] Y. Baskin and L. Meyer, "Lattice constants of graphite at low temperatures," Physical Review, vol. 100, no. 2, p. 544, 1955.

[47] Y. Fujimoto and S. Saito, "Formation, stabilities, and electronic properties of nitrogen defects in graphene," Physical Review B, vol. 84, no. 24, Article ID 245446, 2011.

[48] Y. Fujimoto and S. Saito, "Structure and stability of hydrogen atom adsorbed on nitrogen-doped carbon nanotubes," Journal of Physics: Conference Series, vol. 302, no. 1, Article ID 012006, 2011.

[49] Y. Fujimoto, K. Hirose, and T. Ohno, "Calculations of surface electronic structures by the overbridging boundary-matching method," Surface Science, vol. 586, no. 1-3, pp. 74-82, 2005.
[50] Y. Fujimoto and S. Saito, "Energetics and electronic structures of pyridine-type defects in nitrogen-doped carbon nanotubes," Physica E, vol. 43, no. 3, pp. 677-680, 2011.

[51] Y. Kim, J. Ihm, E. Yoon, and G.-D. Lee, "Dynamics and stability of divacancy defects in graphene," Physical Review BCondensed Matter and Materials Physics, vol. 84, no. 7, Article ID 075445, 2011.

[52] O. V. Yazyev and L. Helm, "Defect-induced magnetism in graphene," Physical Review B-Condensed Matter and Materials Physics, vol. 75, no. 12, Article ID 125408, 2007.

[53] T. O. Wehling, A. V. Balatsky, M. I. Katsnelson, A. I. Lichtenstein, K. Scharnberg, and R. Wiesendanger, "Local electronic signatures of impurity states in graphene," Physical Review B, vol. 75, no. 12, Article ID 125425, 2007.

[54] H. Amara, S. Latil, V. Meunier, P. Lambin, and J.-C. Charlier, "Scanning tunneling microscopy fingerprints of point defects in graphene: a theoretical prediction," Physical Review BCondensed Matter and Materials Physics, vol. 76, no. 11, Article ID 115423, 2007.

[55] K. S. Kim, A. L. Walter, L. Moreschini et al., "Coexisting massive and massless Dirac fermions in symmetry-broken bilayer graphene," Nature Materials, vol. 12, no. 10, pp. 887-892, 2013.

[56] K. S. Novoselove, E. McCann, S. V. Morozov et al., "Unconventional quantum Hall effect and Berry's phase of $2 \pi$ in bilayer graphene," Nature Materials, vol. 12, pp. 887-892, 2013.

[57] Y. Fujimoto and S. Saito, "Electronic structures and stabilities of bilayer graphene doped with boron and nitrogen," Surface Science, vol. 634, pp. 57-61, 2015.

[58] M. G. Menezes, R. B. Capaz, and J. L. B. Faria, "Gap opening by asymmetric doping in graphene bilayers," Physical Review BCondensed Matter and Materials Physics, vol. 82, no. 24, Article ID 245414, 2010.

[59] Y. Fujimoto, T. Koretsune, and S. Saito, "Electronic structures of hexagonal boron-nitride monolayer: strain-induced effects," Journal of the Ceramic Society of Japan, vol. 122, no. 1425, pp. 346-348, 2014.

[60] Y. Fujimoto and S. Saito, "Atomic geometries and electronic structures of hexagonal boron-nitride bilayers under strain," Journal of the Ceramic Society of Japan, vol. 123, no. 1439, pp. 576-578, 2015.

[61] J. Tersoff and D. R. Hamann, "Theory and application for the scanning tunneling microscope," Physical Review Letters, vol. 50, no. 25, pp. 1998-2001, 1983.

[62] J. Tersoff and D. R. Hamann, "Theory of the scanning tunneling microscope," Physical Review B, vol. 31, article 805, 1985.

[63] H. Okada, Y. Fujimoto, K. Endo, K. Hirose, and Y. Mori, "Detailed analysis of scanning tunneling microscopy images of the $\mathrm{Si}(001)$ reconstructed surface with buckled dimers," Physical Review B-Condensed Matter and Materials Physics, vol. 63, no. 19, Article ID 195324, 2001.

[64] Y. Fujimoto, H. Okada, K. Endo, T. Ono, S. Tsukamoto, and K. Hirose, "Images of scanning tunneling microscopy on the $\mathrm{Si}(001)-\mathrm{p}(2 \times 2)$ reconstructed surface," Materials Transactions, vol. 42, no. 11, pp. 2247-2252, 2001.

[65] Y. Fujimoto and A. Oshiyama, "Structural stability and scanning tunneling microscopy images of strained Ge films on $\mathrm{Si}(001)$," Physical Review B-Condensed Matter and Materials Physics, vol. 87, no. 7, Article ID 075323, 2013.

[66] Y. Fujimoto and A. Oshiyama, "Atomic structures and energetics of $90^{\circ}$ dislocation cores in Ge films on $\mathrm{Si}(001)$," Physical Review B, vol. 81, no. 20, Article ID 205309, 5 pages, 2010. 
[67] Y. Fujimoto, H. Okada, K. Inagaki, H. Goto, K. Endo, and K. Hirose, "Theoretical study of scanning tunneling microscopy on Si(001) surface," Japanese Journal of Applied Physics, vol. 42, no. 8, pp. 5267-5268, 2003.

[68] L. Zhao, R. He, K. T. Rim et al., "Visualizing individual nitrogen dopants in monolayer graphene," Science, vol. 333, no. 6045, pp. 999-1003, 2011.

[69] R. Yan, Q. Zhang, W. Li et al., "Determination of graphene work function and graphene-insulator-semiconductor band alignment by internal photoemission spectroscopy," Applied Physics Letters, vol. 101, no. 2, Article ID 022105, 2012.

[70] Y.-J. Yu, Y. Zhao, S. Ryu, L. E. Brus, K. S. Kim, and P. Kim, "Tuning the graphene work function by electric field effect," Nano Letters, vol. 9, no. 10, pp. 3430-3434, 2009.

[71] H. Kim, O. Renault, A. Tyurnina et al., "Doping efficiency of single and randomly stacked bilayer graphene by iodine adsorption," Applied Physics Letters, vol. 105, no. 1, Article ID 011605, 2014. 

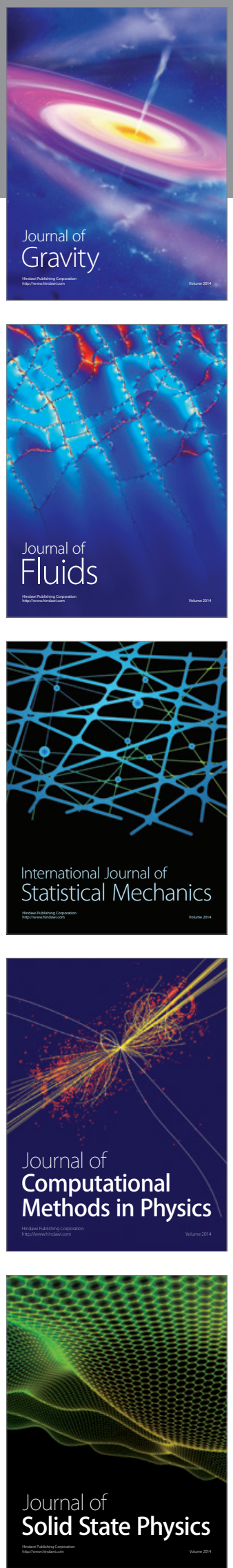

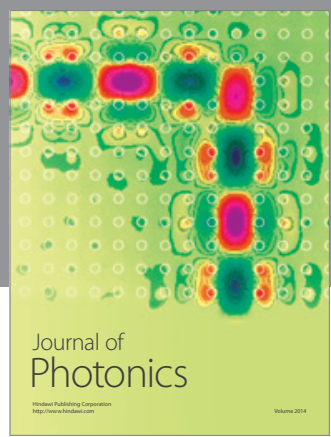

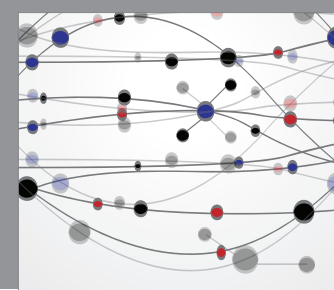

The Scientific World Journal

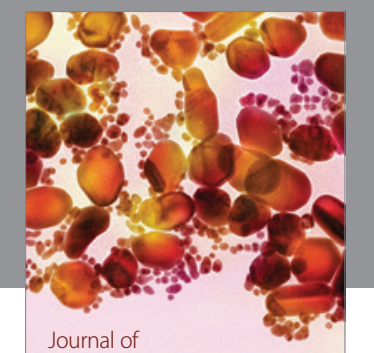

Soft Matter
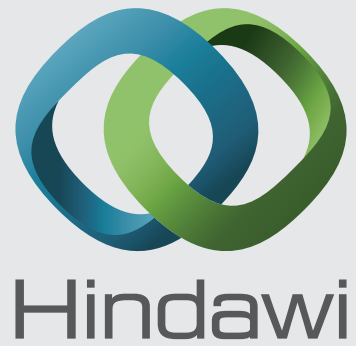

Submit your manuscripts at

http://www.hindawi.com
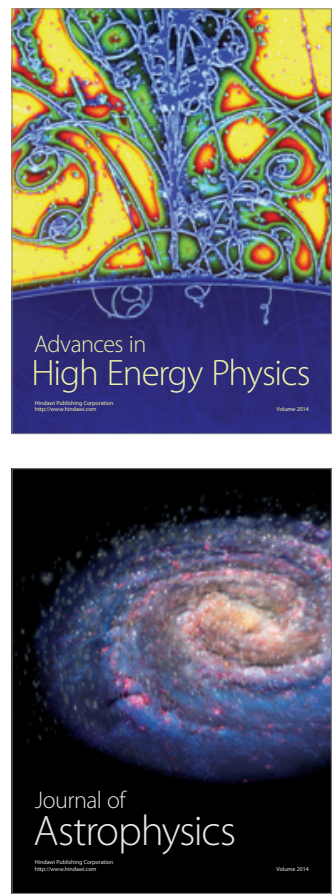
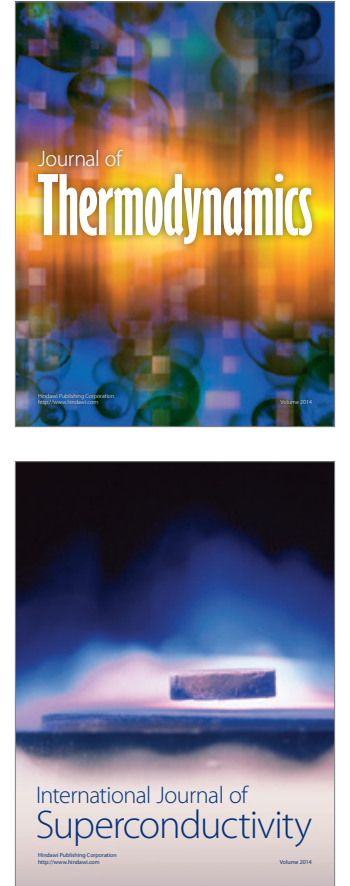
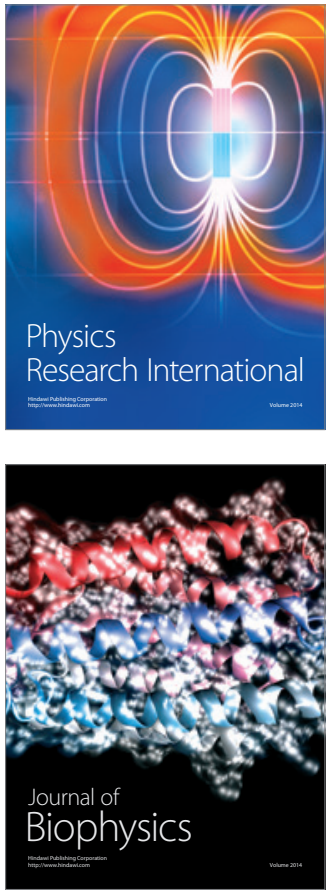
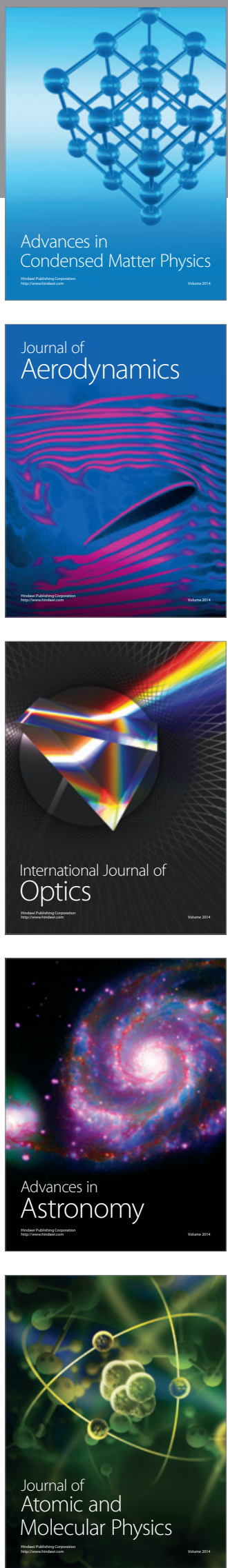